\title{
Prepositional Phrase Attachment Global Ambiguity Resolution in Semantically Biased and Neutral Conditions by L2 Learners
}

\author{
Hadi Maghsoud ${ }^{1, *}$ \\ ${ }^{1}$ Faculty of Foreign Languages and Literatures, University of Tehran, Tehran, Iran \\ *Correspondence: Faculty of Foreign Languages and Literatures, University of Tehran, Tehran, Iran. E-mail: \\ hadi.maghsoud@gmail.com
}

Received: March 12, 2018

Accepted: April 21, 2018 Online Published: June 12, 2018

doi:10.5430/ijelt.v5n2p20

URL: https://doi.org/10.5430/ijelt.v5n2p20

\begin{abstract}
This study investigated the prepositional phrase attachment preferences of Persian speaking second language learners of English in dealing with globally ambiguous sentences. Results are reported from 34 participants across two proficiency levels who took part in an online reading task and an offline reading task. In both tasks, the preferences were examined in biased and neutral conditions; in the former the verb was semantically biased towards the DP inside the PP (e.g. Peter killed the man with a gun) and in the latter condition there was no bias (e.g. Peter met the man with a gun). The findings showed that in both online and offline reading tasks participants resolved the ambiguity by attaching the ambiguous DP to the verb, being influenced by the semantic relationship between the two, but in the neutral condition, the preferences were almost equal for both interpretations. The proficiency level did not influence preferences except for a slight interaction effect in the offline reading task. The findings indicate that L2 learners of different proficiency levels rely on semantics rather than syntax in processing globally ambiguous sentences. The findings of this study in the neutral condition, are in line with the unrestricted race model. However, this model and the constraint-based theories fail to provide a proper explanation for the biased and neutral conditions respectively. It is suggested that discrepancy between the assumptions of the constraint-based theories and the unrestricted race model seems to be due to ignoring the role of semantic relationship between phrases in sentences used for elicitation of parsing preferences; a factor that needs to be taken into account in future studies.
\end{abstract}

Keywords: global ambiguity, parsing, semantic features, semantic relationship, attachment preference

\section{Introduction}

Ambiguity resolution has been an area of investigation to delve into the mystery of processing. Which interpretation of an ambiguous sentence is chosen first and why has attracted psycholinguists for decades. Research in the area of ambiguity resolution divides into two different views about processing. One view supports priority of syntax in initial analysis of ambiguous sentences. The other view considers different sources of information such as semantics, discourse, and context to have a crucial role in adoption of a reading.

As one of the earliest and by far influential theories of language processing, the garden path theory (Frazier \& Rayner, 1982) has been the focus of much research. Syntax is the dominant feature of this theory. Based on this model, the initial analysis is purely syntactic. If the reader's first analysis turns out to be wrong, as in the case of existence of two different readings in ambiguous sentences, a reanalysis is triggered. It is in the second stage that other sources of information such as semantics, context and background information are involved. Minimal attachment and late closure are two offshoots of this theory. According to minimal attachment, for instance, in sentences with $\mathrm{VP}+\mathrm{DP}+\mathrm{PP}$, parsers attach the PP to the verb (high attachment) in their initial analysis. In late closure, the new words are attached to the recently processed phrase or clause.

Although early research was dominated by the role of syntax in processing, it was questioned by advocates of constraint-based theories which highlighted different sources of information such as context, semantics and background knowledge as having an important role in initial parsing. For instance, Cuetos and Mitchell (1988) cast doubt over universality of garden-path theory and late closure, in particular, for all languages. Altmann and Steedman (1988) bolded the determining effect of context through an interaction between syntax and semantics in 
processing. Tanenhaus et al. (1995) provided evidence for the effect of visual context on language processing. Another study indicated the effect of pre-trial pictorial priming of an interpretation on the resolution of ambiguous sentences with prepositional phrases (Branigan, Pickering, \& McLean, 2005). In constraint-based theories, it is assumed that all the syntactic analyses are present simultaneously during processing and " $[t]$ he analysis that receives most support from the various constraints becomes most activated and is selected" (Van Gompel, 2013, p.8).

An amalgamation of the garden path theory and constraint-based theories was proposed as the unrestricted race model (Traxler, Pickering \& Clifton, 1998; Van Gompel, Pickering \& Traxler, 2000). "In this model, processing difficulty is due to reanalysis, but multiple sources of information can determine which analysis is initially adopted" (Van Gompel, Pickering \& Traxler, 2000, p.621).

Compared to the large number of studies on ambiguity resolution by native speakers, only few studies have been conducted on L2 learners. Research on L2 processing has shown that L2 learners, unlike native speakers of English, do not apply phrase structure rules initially to resolve ambiguity (Felser, Roberts, Marinis, \& Gross, 2003) and that monolinguals and bilinguals do not follow the same parsing strategies (Dussias, 2003). Moreover, L2 learners' parsing seems to be more lexically driven and it is highly unlikely that they resort to L1 transfer (Papadopoulou \& Clahsen, 2003). Differences between L1 and L2 processing lead to formation of shallow structure hypothesis (SSH) (Clahsen \& Felser , 2006). According to SSH, processing may take place at two levels. The first level is associated with native speakers at which full engagement of syntax is required. At the second level a partial integration of syntax is required which is supplemented by semantics and other information sources. L2 learners are considered to be second level parsers since their access to syntactic information is limited. However, the results of some studies manifested that highly proficient L2 learners applied processing strategies similar to native speakers (Hopp, 2006; Jackson \& Dussias, 2009; Witzel, Witzel, \& Nicol, 2012).

While a large number of studies have concentrated on processing temporarily ambiguous sentences, only few studies, if any, have been carried out on the way second language learners process globally ambiguous sentences containing prepositional phrases. Moreover, in these studies the role of semantic features of the verb and their overlap with those of the determiner phrase (DP) inside the prepositional phrase (PP) have not been taken into account.

The focus of the present study is attachment preferences of Persian speaking second language learners of English in globally ambiguous sentences in which the way words are grouped together is the source of ambiguity. For example, in the ambiguous sentence the man saw a girl with sunglasses, in one analysis a girl with sunglasses (DP/low attachment) can be grouped together and in another analysis saw with sunglasses (VP/high attachment) and since both analyses are allowed (in the absence of prior knowledge or context), it is globally ambiguous. However, in the absence of extrasentential information, there might be some intrasentential clues such as verb type and definiteness (Spivey-Knowlton \& Sedivy, 1994) and argument structure of the verb (Frenck-Mestre \& Pynte, 1997) which can direct individuals to prefer one attachment over another. To date, it seems, no study has considered the role of shared semantic features of words, specifically semantic overlap between the verb and the DP inside the PP, in ambiguity resolution. For example, in the globally ambiguous sentence above where both interpretations hold true in the absence of context, saw and glasses share a semantic component in that both have a +vision feature in them. This semantic component may act as a clue for readers to attach with sunglasses to the verb rather than to the object DP (i.e. the girl).

The present study utilized globally ambiguous sentences with the structure of VP+DP1+PP(DP2) to determine L2 learners' preference of one reading over another and whether their preference is affected by the semantic relationship between the verb and DP2. If they resort to semantic relationship as a source of information and attach the DP inside the PP (i.e. DP2) to the verb with which it shares semantic features, it means that they do not solely follow a structural analysis. This entails, also, showing a different attachment preference by L2 learners when neutral verbs (with no semantic relationship with the DP inside the PP) are used in the globally ambiguous sentences. However, attaching the DP inside the PP to the verb regardless of presence or absence of semantic relationship between them shows dominance of syntactic or structure-based processing. In this case, L2 learners' preferences would be the same.

The following questions are the focus of the present study.

1. Does the semantic relationship between the verb and the DP inside the PP in globally ambiguous sentences affect PP attachment preferences of Persian speaking second language learners of English across different proficiency levels in the online reading task?

2. Does the semantic relationship between the verb and the DP inside the PP in globally ambiguous English 
sentences affect PP attachment preferences of Persian speaking second language learners of English across different proficiency levels in the offline reading task?

\section{Method}

\subsection{Participants}

This study began with 68 male and female second language learners of English. The participants studied English in private institutes. Their age ranged from 12 to 24 (with mean age of 15.8). All of them spoke Persian as their first language. The participants were naïve to the purpose of the experiment. By the end of the two tasks and the placement test, which were administered in three sessions, the number of participant shrunk to 34 participants. This shrinkage was due to absence of some participants in one of the three sessions of the study. Based on the results of the Oxford Placement Test, those who had attended the three experimentation sessions were divided into two proficiency levels of intermediate and advanced.

\subsection{Instruments}

Two tasks, namely, an online reading task and an offline reading task were used to elicit the participants' preferences.

\subsubsection{Online and Offline Reading Tasks}

For the online and offline reading tasks a total number of 64 (30 experimental and 34 filler) items were developed (See Appendix for the experimental items for the online and offline reading tasks). All the experimental items had the structure $\mathrm{V}+\mathrm{DP} 1+\mathrm{PP}(\mathrm{DP} 2)$ and were ambiguous in that the PP in them could be interpreted as referring to both the verb and the object. The items were grouped into fifteen pairs. Each pair contained a sentence in which the verb had a semantic relationship with DP2 (biased condition) (1a below) and a sentence in which the verb was not semantically biased toward DP2 (neutral condition) (1b below).

(1a) Alex hit the man with a sword.

$$
\mathrm{V} \frac{\mathrm{DP} 1}{\mathrm{DP} 2}
$$

(1b) Alex followed the man with a sword.

$\mathrm{V}$ DP1 DP2

In sentence (1a) the verb hit and the noun sword share some semantic features in that they are both used when talking about injuring someone. But, the verb followed in sentence (1b) does not denote any act of injuring and thus not related to sword.

Each item was followed by two options, each representing a different interpretation of the sentence. For the purpose of illustration, a sample item is provided below:

(2) John threatened the man with a knife.
a) John had a knife
b) The man had a knife

The fillers were developed in a way to be similar in the surface structure to those of experimental items but temporarily ambiguous with a sharp bias toward one interpretation. In other words, there was only one possible answer for a filler. The fillers were expected to distract the participants' attention from the subject under study. Moreover, participants' answers to filler items showed whether the participants were attentive during the tasks and their choices were based on comprehension of the items not a mere chance. An example is provided below:

(3) The spy saw the cop with a gun.

a) The spy had a gun.

b) The cop had a gun.

In half of the items (both experimental and filler) the correct answer was placed first (i.e. choice a) and in the other half it was places second (i.e. choice b). This was done so that after answering a couple of items the participants would not come up with a pattern that, for example, all the $a$ choices are correct.

To avoid ordering effect, all the experimental and filler items were fed to an online randomizer software twice to 
obtain two lists of A and B. Each list contained all the items but with different orderings. Later, almost half of the participants would receive list A and the other half list B. Also, those who received, for example, list A in the online reading task would receive list $\mathrm{B}$ in the offline reading task and vice versa. In other words, the items for the online and offline reading tasks were the same and only the ordering of items varied within and between the two tasks.

Since the online reading task required the participants to answer 64 items under time pressure with focused attention, each list was divided into two halves with each half containing 32 items so that the participants could have break in between. Two fillers were placed at the beginning of the first half to act as a warmup. The same thing was done for the second half (i.e. after the break).

\subsubsection{Item Development}

In the process of item development, four considerations were taken into account. First, it was decided that all the items, both experimental and filler, be similar in structure so that participants fail to form any hypotheses as to the focus of the study. Second, at a point in the process of item development for the online reading time task, it was decided that wh- or yes/no questions be used following experimental items and the participants provide the interpretation as an answer to those questions. However, it was argued that participants' interpretation might be affected by the discourse saliency caused by subjecthood (Cunnings, 2016). For instance, after sentence (4) appeared on the screen, the participants would be required to provide an answer for a question such as (5) written on a paper questionnaire. Here, the participants could rely on their memory to answer. The fact that Eric is a proper noun and the subject of the sentence makes it more salient compared to the man; thus, it is prone to be remembered by the participants leading them to think that a black coat modified Eric. Therefore, the whole idea was abandoned.

(4) Eric met the man with a black coat.

(5) Who was wearing a black coat?

Animacy effect was the third concern, since "animacy affects the choice of syntactic structure" (Fukumura \& van Gompel, 2011). An animate noun "refer[s] to a person, animal, or other creature (boy, sheep, worm), in contrast to an inanimate noun, which refers to a thing or concept (corn, boyhood, sleep)" (McArthur \& McArthur, 1998, p.42). Animate nouns are more accessible than inanimate ones (Aissen, 2003). Therefore, in (6) participants are more likely to perceive Tim (animate) as the more prominent compared to the room (inanimate) and so relate with a picture to it but in (7) where both the man and Eric are animate, with a black coat can be attached to any of them. Accessibility of the subject DP might override the semantic relationship (or lack of relationship) between the verb and the DP inside the PP. To avoid this, both DPs in each sentence were animate (as in 11) in all the experimental items.

(6) Tim decorated the room with a picture.

Animate Inanimate

(7) Eric met the man with a black coat. Animate Animate

Fourth, since the influence of the semantic relationship between the verb and the DP inside the PP was the focus of this study, the object DP had to be written in a way that had no relationship with the DP inside the PP. For example, in (8) the DP inside the PP a gun has a semantic relationship both with the verb arrest and the object DP the burglar.

(8) The cop arrested the burglar with a gun.

To avoid the effect of the semantic features of the object DP on the participants' attachment preference, the DP the man, which is neutral, was written as the object DP in all experimental sentences.

\subsubsection{L1 Transfer}

The impact of first language on L2 learners' processing mechanism has been addressed by many studies (e.g. Havik, Roberts,Van Hout, Schreuder \& Haverkort, 2006; Jackson \& Dussias, 2009; Marinis, Roberts, Felser \& Clahsen, 2005; Roberts, Gullberg \& Indefrey, 2008). Therefore, it is necessary to investigate the possibility of transfer in this study from participants' L1, which is Persian. To address this, a comparison needs to be made between structures of Persian and English sentences which contain grouping ambiguity. An ambiguous English sentence with its Persian translations are presented here.

(16) John arrested the burglar with a gun. 


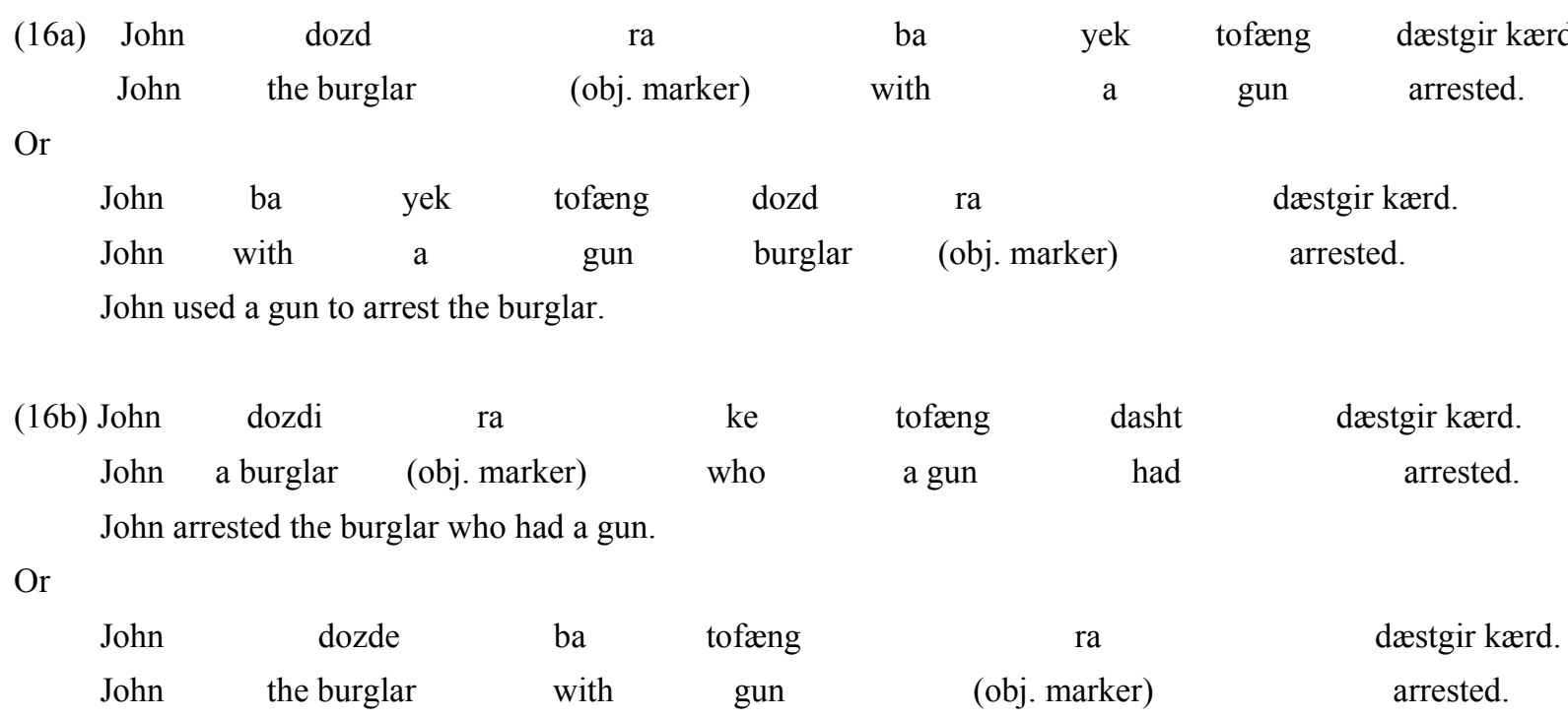

In Persian two different structures are used for the ambiguous English sentence (16). Sentence (16a) which has two structures with the same meaning, is used when it is intended to say that John owned the gun (i.e., with the gun is attached high to the verb, minimal attachment). Sentence (16b) is used when the burglar is carrying the gun. In other words, the PP with the gun is attached to the DP the burglar (low attachment). Obviously, Persian speakers use different unambiguous sentences to refer to the two interpretations of sentence (16). In fact, they do not encounter such an ambiguity in English in their own mother tongue. Therefore, it is highly unlikely that Persian as the participants' first language plays a determinant role in biasing toward either reading this kind of ambiguity.

\subsubsection{Piloting Items}

In developing items for online and offline reading tasks, attempt was made to choose verbs that had a strong semantic relationship with the DP inside the PP (biased verbs) in 15 experimental items. The verbs chosen for the other 15 items were assumed to show no such relationship (unbiased verbs, neutral condition). Therefore, a pilot study was conducted to make sure that the expectations about the semantic relationship or lack of it between the verb and the DP inside the PP were approved by others.

The experimental sentences were piloted in the form of a Likert scale questionnaire to make sure of the assumed semantic relationship between the verb and the DP inside the PP. Thirteen TEFL M.A. students judged the strength of semantic relationship between the verb and the DP inside the PP on a scale of 1 (weak) to 6 (strong).

Regarding the 15 sentences with biased verbs, analysis of the results of the pilot study showed that the participants' judgment met the author's expectation up to 97 percent. In other words, for those sentences, the majority of the participants marked scales 5 and 6 and perceived the DP inside the PP as semantically related to the verb. In 71 percent of the cases, for the 15 sentences with unbiased verbs, scales 1 and 2 were marked, i.e., for the assumed unbiased verbs, the majority of the participants perceived the verb as semantically unrelated to the DP inside the PP. The results of the pilot study seemed to meet the expectations regarding the semantic relationship between the elements in question. A few problematic items that were identified as non-biased by the students were modified.

\subsection{Data Collection Procedure}

Both tasks were administered to learners in groups.

\subsubsection{The Online Reading Task}

The online reading task was administered first. The reason was that if the offline reading task were administered first, the participants could have enough time to think about the items which in turn could affect their attachment preference in the online reading task. Before the main experiment, two individuals with different proficiency levels did the task as a pilot study; no problems were reported.

As the first step in conducting the experiment, each participant received an answer sheet which also contained language background questions and instructions. For background information, they answered questions about their first language, the language spoken at home, number of years spent learning English, and residence in another 
country. Then, they were told to read the instructions written in Persian on the answer sheet. An oral explanation was also given to ensure the participants have understood the task thoroughly.

The task was modeled using five practice items to familiarize the participants with the procedure. The participants marked their preferences in the specified part on the answer sheet. The participants were, then, asked if they had any problems such as the visibility of the sentences, timing, or familiarity with the procedures. No particular problem was reported.

The experimental items were presented on an automated PowerPoint slide show. The items were timed. The participants first saw the stem of each item with its number on top for seven seconds. After that, the stem gave its place to the two choices which stayed on the screen for six seconds. Then, a play sign $\bullet$ appeared lasting for 5 seconds during which participant could mark their preferences in the answer sheet and prepare themselves for the upcoming stem. These times were estimated on the basis of optimal time for reading the item stems and choices and getting ready for the next item. In pilot sessions, individuals were asked if they were comfortable with the timing and no problems were reported. Showing all 64 items took approximately 18 minutes. Since the task demanded participants' constant focused attention during the 18 minutes, to prevent distraction caused by fatigue, the participants were given a short break in the middle of the experiment (after answering 32 of the items).

Regarding the ordering effect, as was explained previously, almost an equal number of participants in different classes saw items in two different orders of A and B.

\subsubsection{The Offline Reading Task}

The second task was conducted approximately three weeks after the online reading task. In the offline reading task, the participants were given questionnaires which contained the same items as the online reading task but with a different ordering. Then, they were asked to read the items in the questionnaire and tick boxes on the answer sheet, which corresponded to their choice of the readings for each item. The difference between the online and offline reading tasks was that in the offline reading task, the participants had the opportunity to think about their judgment since they had more time. To reduce memory effect (i.e. carry-over of the previously met information), the questionnaires were counterbalanced so that the participants who had received ordering $\mathrm{A}$ in the online task this time receive ordering $\mathrm{B}$ and vice versa. All the participants finished the task in less than 30 minutes.

\subsubsection{Placement Test}

After the experiments, and on a different day, the participants took a placement test (UCLES, 2001) to determine their proficiency level. This test included cloze and multiple choice tests which addressed knowledge of grammar, vocabulary and pragmatics in an integrated manner.

\section{Results}

\subsection{Results for the Placement Test}

Before analysis of the data obtained from the tasks of this study, the mean of scores for the placement test which was used to determine learners' level was calculated (23.14). The maximum possible score in the test was 40 . The descriptive statistics for the placements test are illustrated in Table 1.

Table 1. Descriptive Statistics for the Placement Test $(\mathrm{N}=34)$

\begin{tabular}{lcccc}
\hline & Minimum & Maximum & Mean & Std. Deviation \\
\hline Placement test & 16 & 32 & 23.14 & 4.03 \\
\hline
\end{tabular}

Then, the participants were divided to high (i.e. those who received scores above the mean) and low (i.e. those who received scores below the mean) proficiency levels. Nineteen participants fell in the low proficiency level group and fifteen in the high proficiency level group. Table 2. below shows the descriptive statistics for the two groups. Also, the results of an independent samples t-test revealed a statistically significant difference between the two groups ( $\mathrm{t}_{32}$ $=7.604, \mathrm{p}=.000)$. 
Table 2. Descriptive Statistics for Low and High Proficiency Level Groups ( $\mathrm{N}=34$ )

\begin{tabular}{llcc}
\hline Proficiency & N & Mean & Std. Deviation \\
\hline Low & 19 & 20.31 & 2.33 \\
High & 15 & 26.73 & 2.57 \\
\hline
\end{tabular}

\subsection{Results for the Online Reading Task}

For the online reading task, the participants' data that met the criterion of correctly answering 80 percent of the fillers (i.e. 27 out of 34) was considered as acceptable. Since all the scores were above the criterion, no one was excluded from the analysis. Table 3. provides the descriptive statistics for the filler items.

Table 3. Descriptive Statistics for Fillers in the Online Reading Task $(\mathrm{N}=34)$

\begin{tabular}{lcccc}
\hline & Minimum & Maximum & Mean & Std. Deviation \\
\hline Fillers & 28 & 34 & 31.38 & 1.72 \\
\hline
\end{tabular}

The table above shows that the participants with the lowest score received 28 which means they all correctly answered more than 82 percent of the fillers.

The scores from the experimental items were the result of adding up the number of the times an individual showed a verb attachment preference. Therefore, to meet the assumptions of parametric tests, they were transformed to percentage to obtain interval scores. The normality was checked by Shapiro-Wilk. The descriptive statistics for the replies in the biased and neutral conditions across the two proficiency levels are presented in Table 4.

Table 4. Descriptive Statistics for the Replies in the Biased and Neutral Conditions across Two Proficiency Levels in the Online Reading Task $(\mathrm{N}=34)$

\begin{tabular}{lcccc}
\hline & & Attachment to the Verb & $\begin{array}{c}\text { Attachment to the Object } \\
\text { DP }\end{array}$ \\
\hline Condition & Proficiency & Mean & Mean & Std. Deviation \\
\hline \multirow{2}{*}{ Biased } & Low & 81.40 & 18.59 & 13.06 \\
& High & 87.55 & 12.44 & 4.26 \\
\multirow{3}{*}{ Neutral } & Low & 51.92 & 48.07 & 20.07 \\
& High & 48.07 & 54.78 & 22.09 \\
\hline
\end{tabular}

As the means in the table show, in the biased condition, participants in both groups of low (mean=81.40) and high (mean=87.55) proficiency levels opted to attach the DP inside the PP to the verb rather than to the object DP. This is an indication that semantic bias of the verb towards the DP inside the PP affected the participants' preferences. In the neutral condition, however, neither group, with means of 51.92 and 48.07 for low and high proficiency levels respectively, showed any preference between the two options. This means that the participants did not show a special preference for a particular reading in the neutral condition. Figure 1. shows the preferences in biased and neutral conditions across two proficiency levels. 


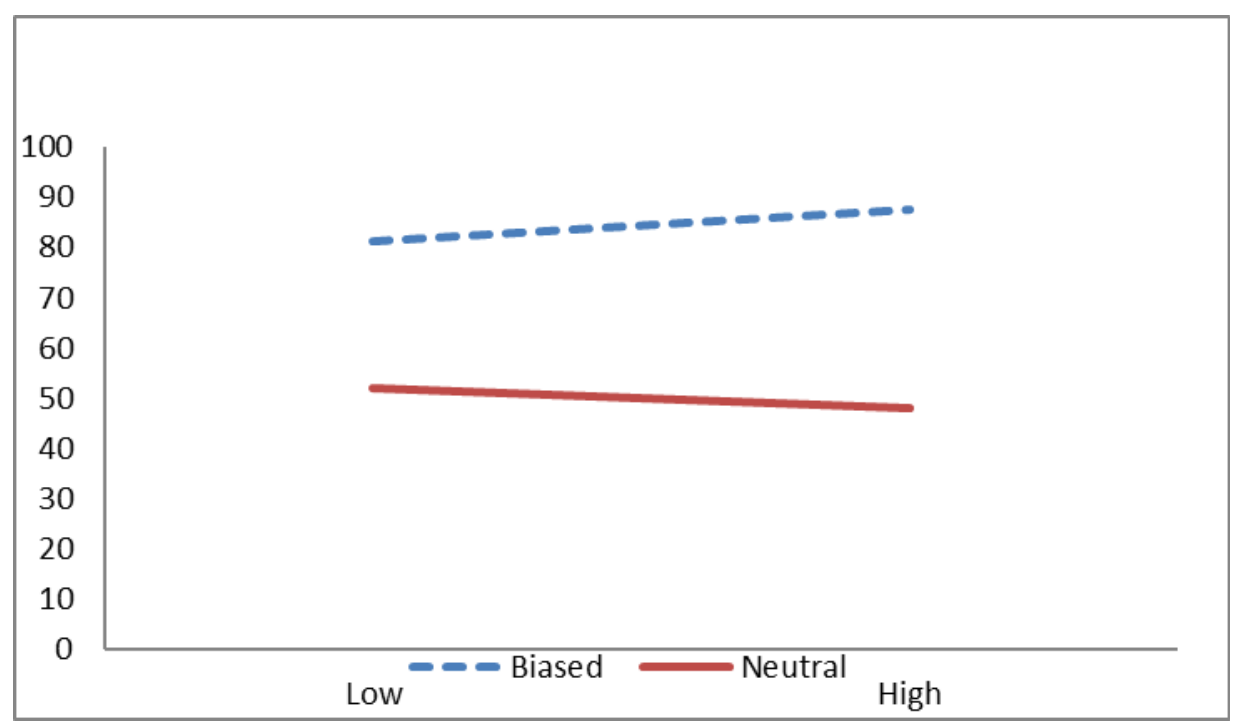

Figure 1. Attachment to the Verb across Different Conditions and Proficiency Levels in the Online Reading Task

To make sure that the mean differences referred to above are statistically significant a $2 \times 2$ repeated measures ANOVA was conducted. The variables were condition as the within-subjects variable and proficiency as the between-subjects variable. The two levels of the condition variable referred to when there was a semantic relationship between the verb and the DP inside the PP (biased condition) and when there was a neutral verb and therefore no such relationship existed (neutral condition). In fact, this analysis investigated the participants' preferences between the condition in which a verb bias existed and the condition in which there was no verb bias. Proficiency as the between-subjects variable had two levels of high and low.

Since the results for responses which were reflective of attaching the DP inside the PP to the verb were complementary to the responses reflective of attaching it to the object DP in each condition, only the analysis of the results for the verb-attached responses are reported.

The results revealed that condition had a significant main effect on attachment preference, $F(1,32)=94.113$, $\mathrm{P}=.000, \mu^{2}=.746$. However, such effect was observed neither for proficiency, $\mathrm{F}(1,32)=.000, \mathrm{P}=1, \mu^{2}=1000$, nor for interaction between condition and proficiency, $\mathrm{F}(1,32)=2.806, \mathrm{P}=.104, \mu^{2}=.081$. As the effect sizes indicate, about 75 percent of variation in responses is accounted for by condition.

According to the results, condition influenced attachment preferences of the participants. However, in none of the biased and neutral conditions did the proficiency level and its interaction with condition have an effect on the participants' attachment preferences.

Moreover, the percentages of attachment to the verb and object DP (Table 4.) indicated that in biased condition where the verb is semantically biased toward the DP inside the PP, the participants preferred to attach the DP inside the PP to the verb rather than the object DP. Nonetheless, in the neutral condition where there is no semantic relationship between the verb and the DP inside the PP, the participants' preferences were approximately equally divided between attachment to the verb and attachment to the object DP.

\subsection{Results for the Offline Reading Task}

Similar to the online reading task, the data from the participants who received scores equal to or higher than 27 out of 34 (higher than 80 percent) was considered as eligible to be used in the analysis. The descriptive statistics for the filler items in the offline task are presented in Table 5.

Table 5. Descriptive Statistics for Fillers in the Offline Reading Task ( $N=34)$

\begin{tabular}{lcccc}
\hline & Minimum & Maximum & Mean & Std. Deviation \\
\hline Fillers & 27 & 34 & 32.00 & 2.18 \\
\hline
\end{tabular}


As is illustrated in the table, all the participants received acceptable scores with the mean of 32 (94.1 percent) regarding the fillers in the offline task.

Similar to the online reading task, the scores were converted to interval scores (i.e. percentages) to satisfy assumptions of parametric tests. The normality was checked using Shapiro-Wilk. Table 6. provides the descriptive statistics for the means of attachment preferences of low and high proficiency level participants in the biased and neutral conditions.

Table 6. Descriptive Statistics for the Replies in the Biased and Neutral Conditions across Two Proficiency Levels in the Offline Reading Task ( $\mathrm{N}=34)$

\begin{tabular}{lcccc}
\hline & \multicolumn{3}{c}{ Attachment to the Verb } & $\begin{array}{c}\text { Attachment to the Object } \\
\text { DP }\end{array}$ \\
\hline Condition & Proficiency & Mean & Mean & Std. Deviation \\
\hline \multirow{2}{*}{ Biased } & Low & 77.89 & 22.10 & 12.18 \\
& High & 82.66 & 17.33 & 11.49 \\
\multirow{3}{*}{ Neutral } & Low & 49.47 & 50.52 & 20.52 \\
& High & 41.33 & 58.66 & 20.65 \\
\hline
\end{tabular}

Table 6. shows a verb attachment mean of 77.89 for low and 82.66 for high proficiency participants in the biased condition which indicates a preference to attach the DP inside the PP to the verb. Therefore, it can be said that verb bias was such a strong force that prevented the participants to attach the DP inside the PP to the object DP in most of the cases. However, in the neutral condition, the fact that the participants almost equally preferred attachment to both the verb and the DP inside the PP shows that they did not have a special tendency for a particular reading. This is indicated by the closeness of means of attachment to the verb and the object DP respectively with the means of 49.47 and 50.52 for low proficiency level and 41.33 and 58.66 for high proficiency level participants.

To ensure that the differences referred to above are statistically significant, a $2 \times 2$ repeated measures ANOVA was performed. In the same way as the online reading task, in this analysis, the within-subjects variable was condition with two levels of biased and neutral (i.e., existence and nonexistence of a semantic relationship between verb and the DP inside the PP) and the between-subjects variable was proficiency which contained two levels of high and low.

It should be noted that the results of the attachment of the DP inside the PP to the verb and to the object DP were complementary in each condition. Therefore, only the analysis of the results for the attachment to the verb are presented.

Similar to the previous task, in the offline reading task, there was a statistically significant main effect of condition on attachment preference, $\mathrm{F}(1,32)=123.342, \mathrm{P}=.000, \mathrm{~m}^{2} \mathbf{m}, \mathrm{m} 4 \mathrm{4}$. The analysis indicated that proficiency, $\mathrm{F}(1,32)=.119, \mathrm{P}=.732, \mathrm{fi}^{2}=1004$ did not significantly influence attachment preferences. However, interaction between condition and proficiency indicated statistically significant main effect, $\mathrm{F}(1,32)=4.226, \mathrm{P}=.048, \mu^{2} \mathbf{m}, 11 \%$. The effect size shows that condition generated approximately 80 percent of variation in replies.

The results show that, in contrast to the considerable effect of condition on the participants' attachment preferences, proficiency did not have a noticeable influence. The interaction between proficiency and condition affected attachment preferences, however, the influence of such interaction was small as evidenced by the very low effect size, i.e., about 12 percent.

Furthermore, based on percentages presented in Table 6., the semantic relationship between the verb and the DP inside the PP strongly influenced the participants' attachment preferences in the biased condition which means they attached the DP inside the PP more to the verb rather than the object DP. In the neutral condition, the replies of the participants did not show that they preferred either attachment to the other. In other words, their preferences were almost evenly balanced between attachment to the verb and to the object DP. In neither condition did the proficiency level show an effect on attachment preferences. However, interaction of proficiency and condition (with the probability of .048) seemed to be influential. This signifies that the members of one proficiency level acted differently from those of the other level. Figure 2. illustrates the interaction of proficiency and condition. 


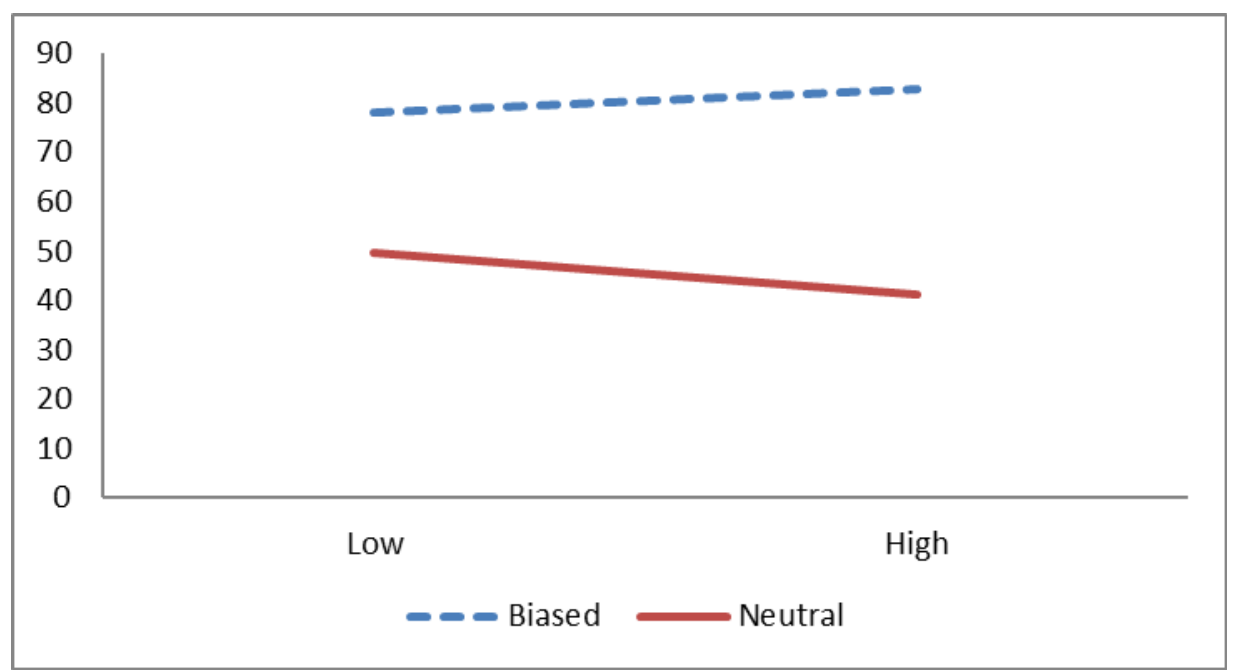

Figure 2. Attachment to the Verb across Different Conditions and Proficiency Levels in the Offline Reading Task

As is shown in the figure, the high proficiency level participants expressed a slightly stronger preference of the low proficiency level participants to attach the DP inside the PP to the verb in the biased condition. In contrast, in the neutral condition, there is a small inclination towards attachment to the object DP at this level of proficiency. In brief, the high proficiency level participants were more sensitive to the semantic relationship between the DP inside the PP and the verb than the low proficiency level participants.

\section{Discussion}

The preferences in different conditions (biased and neutral) and across different proficiency levels in the online reading task were indicative of absence of structure-based parsing by the participants. In the biased condition and at both proficiency levels, the participants' preferences showed to be driven by semantic information in that the preferences were dominantly attachment to the verb rather than the object DP when the verb and the DP inside the PP shared semantic features. In the neutral condition, the comparison of the mean of preferences of low and high proficiency level participants did not indicate the dominance of either semantics or syntax in parsing (refer to Table 4.). Simply put, the L2 learners did not show any specific preference for a particular interpretation.

The results for the offline reading task were almost similar to those of the online reading task. Both high and low proficiency level participants in the biased condition preferred attaching the DP inside the PP to the verb with which it had a semantic relationship. In the neutral condition, neither attachment to the verb nor attachment to the object DP determined the preferences.

However, unlike the online reading task, there was a difference between high and low proficiency level participants in the offline reading task. This was shown by the interaction between condition and proficiency level. In the biased condition, the high proficiency level participants were slightly more inclined than low proficiency participant to attach the DP inside the PP to the verb (refer to Table 6.). However, this trend reversed in the neutral condition, i.e., at high proficiency level, there were more attachments to the object DP. It is worthy to note that the interaction had a large p-value of .048 and a very small effect size of about 12.

Generally, the results for the two tasks indicated that when the verb is semantically biased towards the DP inside the PP (biased condition) in ambiguous sentences, individuals show a tendency to attach the DP inside the PP to the verb. On the surface, it might seem that the participants' parsing is dominated by minimal attachment. However, it must be kept in mind that such a preference is observed only in a condition where the verb is biased. This indicates that L2 learners' parsing preference in globally ambiguous sentences is significantly influenced by semantic cues and not by the syntactic structure. It seems that when confronting a globally ambiguous sentence, between the two interpretations, L2 learners subconsciously go for the one which is semantically supported. The semantic information on the side of the biased verb is so influential that they might not even notice that the sentence could be read in a different way, too. In the same line, Britt (1987, as cited in Britt, Perfetti, Garrod, \& Rayner, 1992) argued that 
sufficiently strong context can overcome syntactic processing. Therefore, based on the findings of this study, sufficiently strong semantic bias can influence L2 learners' preference and lead them away from syntactic parsing.

Different findings for the biased and neutral conditions within the online and offline reading tasks has implications for processing theories. The constraint-based theories and the unrestricted race model assume that the reading is adopted that receives more support. Their difference lies in acceptance of competition. The constraint-based theories assume that individuals experience processing difficulty since both readings are of equal weight and that readings compete until one of them which is supported more is adopted. On the contrary, the unrestricted race model rejects competition between readings and does not predict any reading difficulty. It assumes that preferences for each reading are almost equal. The assumptions of the two theories seem to be at odds. Nevertheless, such difference between theories might be due to presenting a fragmentary account and missing the big picture. For example, in their study to support the unrestricted race model, Van Gompel, Pickering, and Traxler (2001, p.253) compared processing difficulty of globally ambiguous sentences such as (9) and (10) with temporarily ambiguous sentences.

(9) The old man pointed at only the girl with the stick after some time.

(10) The brute frightened only the scoundrel with the penknife late at night.

They concluded that, in contrast to the predictions of the constraint-based theories, "[r]eaders experience[ ] more difficulty when semantic information disambiguated the sentences to NP attachment or VP attachment than when it was consistent with either analysis" (p.225). In other words, globally ambiguous sentences are less difficult to process compared to temporarily ambiguous sentences. They attributed the ease of processing to absence of competition in sentences with interpretations of equal weight. Nevertheless, they seemed to be ignorant of semantic relationship between phrases since they did not take it into account. Although (9) can be interpreted in two meaningful ways, one of the readings enjoys semantic bias of the verb. The verb point is semantically related to the stick; thus, the interpretation which reflects the old man as the holder of the stick is more prominent. Therefore, it can be assumed that less reading difficulty is the result of unequal support for each interpretation. In sentence (10), however, frightened is semantically associated with both scoundrel, and penknife. Thus, both interpretations, i.e. The brute had the knife and the scoundrel had the penknife, are equally plausible. On the surface, the two sentences look similar but investigation of semantic relations of the phrases reveals that the readings of (9) are not as balanced as those of (10). In fact, (9) and (10) resemble the sentences used in the biased and neutral conditions of this study respectively. On that account, difference between assumptions of the constrain-based theories and the unrestricted race model might be due to partial examination of semantic relationships between sentential elements. That is to say that investigation of global ambiguity without exact consideration of the role of semantics results in equivocal outcome.

It seems that the two theories take semantic relations only as neutral for both readings (as in the neutral condition of this study) and that they do not have any specific predictions regarding globally ambiguous sentences where both readings are logically meaningful but one of them is semantically supported (as in the biased condition of this study). In such cases of global ambiguity, less processing difficulty seems to be due to absence of a reanalysis which results from high prominence of one analysis with strong semantic support as in (9). However, processing temporarily ambiguous sentences might entail reanalysis and difficulty in processing.

Proficiency level did not show any significant effect on the participants' attachment preferences in any of the three tasks. In fact, there was almost no indication of differences between high and low proficiency levels. The findings of the present study regarding proficiency level seem to be congruent with shallow structure hypothesis (SSH) (Clahsen \& Felser, 2006). SSH provides a pessimistic account about a late learner of L2 ever becoming native-like regarding parsing. Based on SSH, processing takes place at two levels. The first level that requires full access to syntactic information is only available to the native speakers. L2 learners as shallow parsers, are considered as second level parsers regardless of proficiency level. They compensate for their lack of syntax knowledge by employing other sources of information such as semantics.

\section{Conclusion}

Investigation of attachment preferences of L2 learners across low and high proficiency levels revealed that when neither reading of a globally ambiguous sentence is supported by a semantic bias, there are equal preferences for either reading. When one reading enjoys a semantic support, it is preferred to the one without a semantic support. The proficiency level of the participants did not show to affect attachment preferences except in the offline reading task. In the offline reading task, attachment to the verb preference was slightly stronger at the high proficiency level 
in the biased condition. In the neutral condition, however, such preference was stronger at low proficiency level. In sum, the findings of the current study indicated that semantic relationship between certain elements in globally ambiguous sentences is a determining factor for adoption of a particular reading. Therefore, it is suggested that studies on ambiguity resolution consider the semantic relationship and potential bias of sentential elements. Moreover, the findings of the present study could contribute to translation of globally ambiguous sentences such as those used in this study. The semantic relationship between elements in the sentence or its absence can guide translators to provide an appropriate equivalence of the source language utterance in the target language.

\section{References}

Aissen, J. (2003). Differential object marking: Iconicity vs. economy. Natural Language \& Linguistic Theory, 21(3), 435-483. https://doi.org/10.1023/A:1024109008573

Altmann, G., \& Steedman, M. (1988). Interaction with context during human sentence processing. Cognition, 30(3), 191-238. https://doi.org/10.1016/0010-0277(88)90020-0

Branigan, H. P., Pickering, M. J., \& McLean, J. F. (2005). Priming prepositional-phrase attachment during comprehension. Journal of Experimental Psychology: Learning, Memory, and Cognition, 31(3), 468-481. https://doi.org/10.1037/0278-7393.31.3.468

Britt, M. A., Perfetti, C. A., Garrod, S., \& Rayner, K. (1992). Parsing in discourse: context effects and their limits. Journal of Memory and Language, 31(3), 293-314. https://doi.org/10.1016/0749-596X(92)90015-P

Clahsen, H., \& Felser, C. (2006). How native-like is non-native language processing? TRENDS in Cognitive Sciences, 10(12), 564-570. https://doi.org/10.1016/j.tics.2006.10.002

Cuetos, F., \& Mitchell, D. C. (1988). Cross-linguistic differences in parsing: Restrictions on the use of the Late Closure strategy in Spanish. Cognition, 30(1), 73-105. https://doi.org/10.1016/0010-0277(88)90004-2

Cunnings, I. (2016). Parsing and working memory in bilingual sentence processing. Bilingualism: Language and Cognition, 20(4), 659-678. https://doi.org/10.1017/S1366728916000675

Dussias, P. E. (2003). Syntactic ambiguity resolution in L2 learners: Some effects of bilinguality on L1 and L2 processing strategies. Studies in Second Language Acquisition, 25(4), 529-557. https://doi.org/10.1017/S0272263103000238

Felser, C., Roberts, L., Marinis, T., \& Gross, R. (2003). The processing of ambiguous sentences by first and second language learners of English. Applied Psycholinguistics, 24(3), 453-489. https://doi.org/10.1017/S0142716403000237

Frazier, L., \& Rayner, K. (1982). Making and correcting errors during sentence comprehension: Eye movements in the analysis of structurally ambiguous sentences. Cognitive Psychology, 14(2), 178-210. https://doi.org/10.1016/0010-0285(82)90008-1

Frenck-Mestre, C., \& Pynte, J. (1997). Syntactic ambiguity resolution while reading in second and native languages. Quarterly Journal of Experimental Psychology, 50A, 119-148. https://doi.org/10.1080/027249897392251

Fukumura, K., \& van Gompel, R. P. G. (2011). The effect of animacy on the choice of referring expression. Language and Cognitive Processes, 26(10), 1472-1504. https://doi.org/10.1080/01690965.2010.506444

Havik, E., Roberts, L., van Hout, R., Schreuder, R., \& Haverkort, M. (2009). Processing subject-object ambiguities in the L2: A self-paced reading study with German L2 learners of Dutch. Language Learning, 59(1), 73-112. https://doi.org/10.1111/j.1467-9922.2009.00501.x

Hopp, H. (2006). Syntactic features and reanalysis in near-native processing. Second Language Research, 22(3), 369-397. https://doi.org/10.1191/0267658306sr272oa

Jackson, C. N., \& Dussias, P. E. (2009). Cross-linguistic differences and their impact on L2 sentence processing. Bilingualism: Language and Cognition, 12(1), 65 - 82. https://doi.org/10.1017/S1366728908003908

Marinis, T., Roberts, L., Felser, C., \& Clahsen, H. (2005). Gaps in second language processing. Studies in Second Language Acquisition, 27(1), 53-78. https://doi.org/10.1017/S0272263105050035

McArthur, T., \& McArthur R. (1998). Concise Oxford Companion to the English Language, Oxford University Press. Retrieved April 27, 2017, from http://www.encyclopedia.com/humanities/encyclopedias-almanacs-transcripts-and-maps. 
Papadopoulou, D., \& Clahsen, H. (2003). Parsing strategies in L1 and L2 sentence processing: A study of relative clause attachment in Greek. Studies in Second Language Acquisition, 25(4), 501-528. https://doi.org/10.1017/S0272263103000214

Roberts, L., Gullberg, M., \& Indefrey, P. (2008). On-line pronoun resolution in L2 discourse: L1 influence and general learner effects. Studies in Second Language Acquisition, 30(3), 333-357. https://doi.org/10.1017/S0272263108080480

Spivey-Knowlton, M., \& Sedivy, J. C. (1994). Resolving attachment ambiguities with multiple constraints. Cognition, 55(3), 227-267. https://doi.org/10.1016/0010-0277(94)00647-4

Tanenhaus, M. K., Spivey-Knowlton, M. J., Eberhard, K. M., \& Sedivy, J. C. (1995). Integration of visual and linguistic information in spoken language comprehension. Science, 268(5217), 1632-1634. https://doi.org/10.1126/science. 7777863

Traxler, M. J., Pickering, M. J., \& Clifton, C. (1998). Adjunct attachment is not a form of lexical ambiguity resolution. Journal of Memory and Language, 39(4), 558-592. https://doi.org/10.1006/jmla.1998.2600

Van Gompel, R. P. G., Pickering, M. J., \& Traxler, M. J. (2000). Unrestricted race: A new model of syntactic ambiguity resolution. In A. Kennedy, R. Radach, D. Heller, \& J. Pynte (Eds.), Reading as a perceptual process (pp. 621-648), Oxford: Elsevier. https://doi.org/10.1016/B978-008043642-5/50029-2

Van Gompel, R. P. G., Pickering, M. J., \& Traxler, M. J. (2001). Reanalysis in sentence processing: Evidence against current constraint-based and two-stage models. Journal of Memory and Language, 45(2), 225-258. https://doi.org/10.1006/jmla.2001.2773

Van-Gompel, R. P. G. (Ed.) (2013). Sentence processing, current issues in the psychology of language. London: Psychology Press.

Witzel, J., Witzel, N., \& Nicol, J. (2012). Deeper than shallow: Evidence for structure-based parsing biases in second-language sentence processing. Applied Psycholinguistics, 33(2), 419-456. https://doi.org/10.1017/S0142716411000427

\section{Appendix}

Experimental Items for the Online and Offline Reading Tasks

1. Peter met the man with a gun.

2. Frank hit the man with a walking stick.

3. Andy shot the man with a gun.

4. Alex followed the man with a sword.

5. Sam frightened the man with a Dracula mask.

6. Ben punished the man with a bad manner.

7. Mike threatened the man with a knife.

8. Sara called the man with a cellphone.

9. Eric met the man with a black coat.

10. Andy searched the man with a gun.

11. Ben motivated the man with a bad manner.

12. Bob protected the man with a shield.

13. Sam met the man with a Dracula mask.

14. Alex hit the man with a sword.

15. Frank mimicked the man with a walking stick.

16. Bob left the man with a shield.

17. Roger hit the man with sunglasses.

18. Peter killed the man with a gun. 
19. Carl helped the man with an expensive car.

20. Brad touched the man with a microphone.

21. Tim saved the man with a life jacket.

22. Carl chased the man with an expensive car.

23. Tim followed the man with a life jacket.

24. Carla surprised the man with a gift.

25. Roger saw the man with sunglasses.

26. Eric covered the man with a black coat.

27. Mike approached the man with a knife.

28. Brad talked to the man with a microphone.

29. Sara attacked the man with a cellphone.

30. Carla met the man with a gift. 